\title{
Weighted a priori estimates for elliptic equations
}

\author{
María E. Cejas \\ Departamento de Matemática \\ Facultad de Ciencias Exactas \\ Universidad Nacional de La Plata \\ CONICET
}

Calle 50 y 115, 1900 La Plata, Buenos Aires, Argentina

E-mail: mec.eugenia@gmail.com

Ricardo G. Durán

Departamento de Matemática

Facultad de Ciencias Exactas y Naturales

Universidad de Buenos Aires

IMAS-CONICET-UBA

Pabellón I, Ciudad Universitaria, 1428, CABA, Argentina

E-mail:rduran@dm.uba.ar

\begin{abstract}
We give a simpler proof of the a priori estimates obtained in [DST1, DST2] for solutions of elliptic problems in weighted Sobolev norms when the weights belong to the Muckenhoupt class $A_{p}$. The argument is a generalization to bounded domains of the one used in $\mathbb{R}^{n}$ to prove the continuity of singular integral operators in weighted norms.

In the case of singular integral operators it is known that the $A_{p}$ condition is also necessary for the continuity. We do not know whether this is also true for the a priori estimates in bounded domains but we are able to prove a weaker result when the operator is the Laplacian or a power of it. We prove that a necessary condition is that the weight belongs to the local $A_{p}$ class.
\end{abstract}

2010 Mathematics Subject Classification: Primary 35B45; Secondary 42B20. Key words and phrases: Elliptic equations, weighted a priori estimates. 


\section{Introduction}

The goal of this paper is to prove weighted a priori estimates for solutions of linear elliptic problems with Dirichlet boundary conditions. More precisely, for a bounded smooth domain $\Omega \subset \mathbb{R}^{n}$ we consider

$$
\left\{\begin{aligned}
\mathcal{L} u & =f \text { in } \Omega \\
\mathcal{B}_{j} u & =0 \text { on } \partial \Omega, \quad 1 \leq j \leq m-1
\end{aligned}\right.
$$

where $\mathcal{L}$ is an elliptic operator of order $2 m$ and $\mathcal{B}_{j}$ differential operators of order $m_{j}$ satisfying the properties introduced in the classic paper [ADN].

For $1<p<\infty$, the a priori estimate

$$
\|u\|_{W^{2 m, p}(\Omega)} \leq C\|f\|_{L^{p}(\Omega)}
$$

is well-known. This result is usually referred as Agmon-Douglis-Nirenberg estimate because it is essentially contained in [ADN] although it is not explicitly written in this way in that paper. For completeness we give more details on this point in the next section.

The estimate (1.1) has been extended to weighted norms when $\mathcal{L}$ is the Laplacian or a power of it in [DST1, DST2].

By a weight function we mean a locally integrable function $w$ defined in $\mathbb{R}^{n}$ and for $1 \leq p<\infty$, we define the Banach space $L_{w}^{p}(\Omega)$ with norm given by

$$
\|f\|_{L_{w}^{p}(\Omega)}=\left(\int_{\Omega}|f(x)|^{p} w(x) d x\right)^{1 / p} .
$$

and $W_{w}^{2 m, p}(\Omega)$ the Sobolev space of functions in $L_{w}^{p}(\Omega)$ with derivatives up to order $2 m$ in $L_{w}^{p}(\Omega)$ with the usual norm. For $\Omega=\mathbb{R}^{n}$ we write simply $L_{w}^{p}$ instead of $L_{w}^{p}\left(\mathbb{R}^{n}\right)$. With $C$ we will denote a generic constant which can change its value even in the same line.

For $1<p<\infty$, a weight is in the Muckenhoupt class $A_{p}$ if

$$
\left(\frac{1}{|Q|} \int_{Q} w\right)\left(\frac{1}{|Q|} \int_{Q} w^{-\frac{1}{p-1}}\right)^{p-1} \leq C
$$

for all cubes $Q$. It is well known that the Hardy-Littlewood maximal operator is bounded in $L_{w}^{p}$ if and only if $w \in A_{p}$ (see for example [D]).

For $\mathcal{L}=(-\Delta)^{m}$ it was proved in [DST2] (extending the results for $m=1$ given in [DST1]) that, if $w$ is in $A_{p}$, then

$$
\|u\|_{W_{w}^{2 m, p}(\Omega)} \leq C\|f\|_{L_{w}^{p}(\Omega)},
$$


In this paper we give a different proof of (1.3) generalizing to a bounded domain the classic arguments used to obtain the continuity of singular integral operators in $L_{w}^{p}$. The advantage of this proof is that it is simpler and it does not require estimates of derivatives of the Green function involving the distance to the boundary. On the other hand, our arguments are very general and apply to the class of operators considered in [ADN, K].

We do not know whether the $A_{p}$ condition is also necessary to have (1.3) but we prove a weaker result for the case of $\mathcal{L}=(-\Delta)^{m}$. Indeed, in order to have the a priori estimate $(1.3)$ it is necessary that $w \in A_{p}^{\text {loc }}(\Omega)$ (see below for the definition of this class).

\section{$2 \quad$ Weighted a priori estimates}

For a bounded domain $\Omega \subset \mathbb{R}^{n}$ we consider the problem

$$
\begin{aligned}
& \mathcal{L} u(x):=\sum_{0 \leq|\alpha| \leq 2 m} a_{\alpha}(x) D^{\alpha} u(x)=f(x) \quad \text { in } \Omega \\
& \mathcal{B}_{j} u(x):=\sum_{0 \leq|\alpha| \leq m_{j}} b_{j \alpha}(x) D^{\alpha} u(x)=0 \quad \text { on } \partial \Omega 1 \leq j \leq m-1
\end{aligned}
$$

where $\mathcal{L}$ is uniformly elliptic and $\mathcal{B}_{j}$ satisfy the complementing conditions in the sense of $[\mathrm{ADN}]$.

Our arguments are based on the estimates for the Green function of (2.1) proved in $[\mathrm{K}]$, and therefore, we assume the hypotheses of that paper. Namely, $m_{j} \leq 2 m-1$ and, for $\ell_{0}:=\max _{j}\left(2 m-m_{j}\right)$, let $\ell_{1}$ be an integer such that

$$
\begin{aligned}
& \ell_{1} \geq \frac{3}{2} \ell_{0}, \quad \text { for } n \geq 3 \\
& \ell_{1} \geq 2\left(\ell_{0}+1\right), \quad \text { for } n=2 .
\end{aligned}
$$

Then,

$$
a_{\alpha} \in C^{\ell_{1}+1}(\Omega) \quad b_{j \alpha} \in C^{\ell_{1}+1}(\partial \Omega)
$$

and

$$
\partial \Omega \in C^{\ell_{1}+2 m+1}
$$

Under these assumptions, the existence of the Green function as well as estimates for it and its derivatives were proved in $[\mathrm{K}]$. We state in the next theorem the estimates that we are going to use in our arguments. 
Theorem 2.1. Under the hypotheses (2.2), (2.3) and (2.4) there exists the Green function $G$ of (2.1), namely, the solution $u$ is given by

$$
u(x)=\int_{\Omega} G(x, y) f(y) d y .
$$

Moreover, for $0<\alpha<1$, there exists a constant $C$ depending on $\mathcal{L}, \mathcal{B}_{j}, \Omega$ and $n$ such that

$|\gamma| \leq 2 m \quad, \quad 2 m-n-|\gamma| \neq 0 \quad \Longrightarrow \quad\left|D_{x}^{\gamma} G(x, y)\right| \leq C\left\{|x-y|^{2 m-n-|\gamma|}+1\right\}$

$$
2 m-n-|\gamma|=0 \quad \Longrightarrow \quad\left|D_{x}^{\gamma} G(x, y)\right| \leq C\{|\log | x-y||+1\}
$$

$|\gamma|=2 m \Longrightarrow\left|D_{x}^{\gamma} G(y, z)-D_{x}^{\gamma} G(x, z)\right| \leq C|y-x|^{\alpha}\left(|y-z|^{-n-\alpha}+|x-z|^{-n-\alpha}\right)$.

Proof. See Theorem 3.3 and its Corollary in [K, Page 965].

Our proof of the weighted a priori estimates makes use of the classic unweighted results. As we mentioned in the introduction, the a priori estimate (1.1) is essentially contained in $[\mathrm{ADN}]$ although not written explicitly there. Indeed, in the particular case of homogeneous boundary conditions that we are considering, Theorem 15.2 in page 704 of [ADN] says that

$$
\|u\|_{W^{2 m, p}(\Omega)} \leq C\left\{\|f\|_{L^{p}(\Omega)}+\|u\|_{L^{p}(\Omega)}\right\} .
$$

But, in view of the representation (2.5) and the bound for $G$ given in (2.6), a standard application of the Young inequality yields $\|u\|_{L^{p}(\Omega)} \leq$ $C\|f\|_{L^{p}(\Omega)}$, and therefore, (1.1) follows.

Let us remark that in the above mentioned Theorem 15.2 of [ADN] the authors assume that the norm on the left hand side of the estimate is finite, but this follows from their previous Theorem 7.3 (see also Remark 1 after that theorem) [ADN, Page 668] provided $f$ is regular enough and using again the representation (2.5) to bound the norm of $u$ appearing in the right hand side of that theorem. Then, in the general case one can proceed by a standard density argument.

Let us now recall the argument used in the case of singular integral operators that we are going to generalize. We will make use of the HardyLittlewood maximal operator

$$
\mathcal{M} f(x)=\sup _{Q \ni x} \frac{1}{|Q|} \int_{Q}|f(y)| d y
$$


and of the sharp maximal operator

$$
\mathcal{M}^{\#} f(x)=\sup _{Q \ni x} \frac{1}{|Q|} \int_{Q}\left|f(y)-f_{Q}\right| d y
$$

where the supremums are taken over all cubes containing $x$ and $f_{Q}:=$ $\frac{1}{|Q|} \int_{Q} f$.

If

$$
T f(x)=\lim _{\varepsilon \rightarrow 0} \int_{|x-y|>\varepsilon} K(x, y) f(y) d y
$$

is a singular integral operator which is continuous in $L^{p}$, for $1<p<\infty$, and $K(x, y)$ satisfies

$$
|K(x, z)-K(y, z)| \leq \frac{C|x-y|^{\alpha}}{|x-z|^{n+\alpha}}, \quad \text { for } \quad|x-z| \geq 2|x-y|
$$

with $0<\alpha<1$, then we have, for any $s>1$,

$$
\mathcal{M}^{\#} T f(x) \leq C\left(\mathcal{M}|f|^{s}(x)\right)^{1 / s} .
$$

This estimate is well-known and its proof can be found in several books, although the hypotheses on the operator are not stated usually as we are doing here. Indeed, the proof given in [D, Lemma 7.9] only uses the hypotheses given above.

In the next lemma we prove a version of (2.9) in a bounded domain. With this goal we introduce the local sharp maximal operator

$$
\mathcal{M}_{\Omega}^{\#} f(x)=\sup _{\Omega \supset Q \ni x} \frac{1}{|Q|} \int_{Q}\left|f(y)-f_{Q}\right| d y .
$$

Lemma 2.2. Let $u$ be the solution of (2.1) and assume that the hypotheses of Theorem 2.1 are satisfied. If $|\gamma|=2 m$ we have, for any $s>1$ and any $x \in \Omega$,

$$
\mathcal{M}_{\Omega}^{\#}\left(D^{\gamma} u\right)(x) \leq C\left(\mathcal{M}|f|^{s}\right)^{1 / s}(x)
$$

Proof. We extend $f$ by zero outside of $\Omega$. Let $Q \subset \Omega$ be a cube such that $x \in Q$ and $Q^{*}$ an expanded cube of $Q$ by a factor 2. We decompose $f=$ $f_{1}+f_{2}$ with $f_{1}=f \chi_{Q^{*}}$ and call $u_{1}$ and $u_{2}$ the solutions of (2.1) with $f_{1}$ and $f_{2}$ as right hand sides respectively.

It is known that we can replace $f_{Q}$ by any constant. We choose $a=$ $D^{\gamma} u_{2}(x)$. Then,

$\frac{1}{|Q|} \int_{Q}\left|D^{\gamma} u(y)-a\right| d y \leq \frac{1}{|Q|} \int_{Q}\left|D^{\gamma} u_{1}(y)\right| d y+\frac{1}{|Q|} \int_{Q}\left|D^{\gamma} u_{2}(y)-D^{\gamma} u_{2}(x)\right| d y=(i)+(i i)$. 
Now, given $s>1$, using the Hölder inequality and (1.1), we have

$$
\begin{aligned}
(i) & \leq\left(\frac{1}{|Q|} \int_{Q}\left|D^{\gamma} u_{1}(y)\right|^{s} d y\right)^{1 / s} \leq C\left(\frac{1}{|Q|} \int_{\Omega}\left|f_{1}(y)\right|^{s} d y\right)^{1 / s} \\
& =C\left(\frac{1}{|Q|} \int_{Q^{*}}|f(y)|^{s} d y\right)^{1 / s} \leq C\left(\mathcal{M}|f|^{s}\right)^{1 / s}(x) .
\end{aligned}
$$

On the other hand, if $x \notin \operatorname{supp} f_{2}$ we can take the derivative inside the integral in the expression for $u_{2}$ given by (2.5). Then, since supp $f_{2} \subset\left(Q^{*}\right)^{c}$, for $x \in Q$ we have

$$
(i i) \leq \frac{1}{|Q|} \int_{Q} \int_{\left(Q^{*}\right)^{c}}\left|D_{x}^{\gamma} G(y, z)-D_{x}^{\gamma} G(x, z)\right|\left|f_{2}(z)\right| d z d y .
$$

Therefore, using (2.8) and that $|y-z| \sim|x-z|$ and $|x-z| \geq \frac{\ell(Q)}{2}$ we obtain

$$
\begin{aligned}
(i i) & \leq C \frac{l(Q)^{\alpha}}{|Q|} \int_{Q} \int_{\left(Q^{*}\right)^{c}} \frac{|f(z)|}{|x-z|^{n+\alpha}} d z d y \leq C l(Q)^{\alpha} \int_{\left(Q^{*}\right)^{c}} \frac{|f(z)|}{|x-z|^{n+\alpha}} d z \\
& \leq C l(Q)^{\alpha} \sum_{k=0}^{\infty} \int_{2^{k-1} l(Q)<|z-x|^{\prime} 2^{k} l(Q)} \frac{|f(z)|}{|x-z|^{n+\alpha}} d z \leq C \mathcal{M} f(x)
\end{aligned}
$$

where the last inequality follows by a standard argument (see [D, Lemma 7.9] for details).

The following Lemma is a slightly modified version of [DRS, Theorem 5.23] because we are using a different definition of the sharp maximal operator. The reader can easily check that the proof given in that paper applies to our case.

Lemma 2.3. For $f \in L_{l o c}^{1}(\Omega)$ and $w \in A_{p}$, if $f_{\Omega}=\frac{1}{|\Omega|} \int_{\Omega}|f|$, then

$$
\left\|f-f_{\Omega}\right\|_{L_{w}^{p}(\Omega)} \leq C\left\|\mathcal{M}_{\Omega}^{\#} f\right\|_{L_{w}^{p}(\Omega)}
$$

Now we can state and prove the main result of this section.

Theorem 2.4. Let $u$ be the solution of (2.1) and assume that the hypotheses of Theorem 2.1 are satisfied. Then, for $1<p<\infty, w \in A_{p}$ and $f \in L_{w}^{p}(\Omega)$, there exists a constant $C$ depending on $\mathcal{L}, \mathcal{B}_{j}, \Omega, n$ and $w$ such that

$$
\|u\|_{W_{w}^{2 m, p}(\Omega)} \leq C\|f\|_{L_{w}^{p}(\Omega)}
$$


Proof. If $2 m-n<|\gamma|<2 m$, using (2.5) and (2.6) we obtain

$$
\begin{aligned}
\left|D^{\gamma} u(x)\right| & =\left|\int_{\Omega} D_{x}^{\gamma} G(x, y) f(y) d y\right| \leq C \int_{\Omega} \frac{|f(y)|}{|x-y||\gamma|+n-2 m} d y \\
& \leq C \sum_{k=0}^{\infty} \int_{2^{-(k+1)} d<|x-y| \leq 2^{-k} d} \frac{|f(y)|}{|x-y|^{|\gamma|+n-2 m}} d y \leq C \mathcal{M} f(x)
\end{aligned}
$$

where $d$ denotes the diameter of $\Omega$. For $|\gamma| \leq 2 m-n$ we obtain the same estimate using now that, in view(2.6) and (2.7), for any $\varepsilon>0,|G(x, y)| \leq$ $C|x-y|^{-\varepsilon}$.

Consequently, it follows from the boundedness of the maximal operator for $A_{p}$ weights that

$$
\|u\|_{W_{w}^{2 m-1, p}(\Omega)} \leq C\|f\|_{L_{w}^{p}(\Omega)} .
$$

It rests to estimate $D^{\gamma} u$ for $|\gamma|=2 m$ which is the most difficult part. From Lemmas 2.3 and 2.2 we have

$$
\begin{aligned}
\int_{\Omega}\left|D^{\gamma} u(x)-\left(D^{\gamma} u\right)_{\Omega}\right|^{p} w(x) d x & \leq C \int_{\Omega}\left|\mathcal{M}_{\Omega}^{\#}\left(D^{\gamma} u\right)(x)\right|^{p} w(x) d x \\
& \leq C \int_{\Omega}\left(\mathcal{M}|f|^{s}(x)\right)^{p / s} w(x) d x
\end{aligned}
$$

But it is known that there exists $s$ depending only on $w$ such that $1<s<p$ and $w \in A_{p / s}$ (see for example $[\mathrm{D}]$ ), and using the boundedness of $\mathcal{M}$ in $L_{w}^{p / s}$ we obtain from $(2.11)$,

$$
\int_{\Omega}\left|D^{\gamma} u(x)-\left(D^{\gamma} u\right)_{\Omega}\right|^{p} w(x) d x \leq C \int_{\Omega}|f(x)|^{p} w(x) d x .
$$

Then,

$$
\int_{\Omega}\left|D^{\gamma} u(x)\right|^{p} w(x) d x \leq C\left(\int_{\Omega}|f(x)|^{p} w(x) d x+\int_{\Omega}\left|\left(D^{\gamma} u\right)_{\Omega}\right|^{p} w(x) d x\right)
$$

and so it only remains to estimate the last term. But, since $w$ is integrable in $\Omega$, it is enough to show that

$$
\left|\left(D^{\gamma} u\right)_{\Omega}\right| \leq C\|f\|_{L_{w}^{p}(\Omega)} .
$$

Taking $1<s<p$ and using the a priori estimate (1.1) for $s$ we have

$$
\begin{aligned}
\left|\left(D^{\gamma} u\right)_{\Omega}\right| \leq\left(\frac{1}{|\Omega|} \int_{\Omega}\left|\left(D^{\gamma} u\right)_{\Omega}\right|^{s} d x\right)^{\frac{1}{s}} & \leq C\left(\frac{1}{|\Omega|} \int_{\Omega}|f(x)|^{s} d x\right)^{\frac{1}{s}} \\
& \leq C\left(\frac{1}{|\Omega|} \int_{\Omega}|f(x)|^{p} w(x) d x\right)^{\frac{1}{p}}\left(\frac{1}{|\Omega|} \int_{\Omega} w(x)^{-\frac{s}{p-s}} d x\right)^{\frac{p-s}{s p}}
\end{aligned}
$$


where we have used the Hölder inequality first with $s$ and then with $p / s$. Then, choosing $s$ such that $w \in A_{p / s}$ the last term on the right hand side is finite, and therefore, (2.12) is proved.

\section{$3 \quad$ Necessary condition}

It is known that the $A_{p}$ condition is also necessary for the continuity of singular integral operators [S]. Then, it is natural to ask whether the same is true for the weighted a priori estimates. We do not know the answer but we prove in this section a weaker result, namely, a necessary condition to have the weighted a priori estimates for $\mathcal{L}=(-\Delta)^{m}$ is that the weight belong to the $A_{p}^{l o c}(\Omega)$ class extensively studied in [HSV].

In this section it is more convenient to work with balls instead of cubes. To recall the definition of the $A_{p}^{\text {loc }}$ class, first we consider $0<\beta<1$ and define the $A_{p}^{\beta}(\Omega)$ class as follows. A weight $w$ belongs to this class if

$$
\left(\frac{1}{|B|} \int_{B} w\right)\left(\frac{1}{|B|} \int_{B} w^{-\frac{1}{p-1}}\right)^{p-1} \leq C
$$

for all balls $B \subset \Omega$ such that $\operatorname{diam}(B)<\beta \operatorname{dist}(B, \partial \Omega)$. It was proved in [HSV] that the classes $A_{p}^{\beta}(\Omega)$ are independent of $\beta$, namely, if $0<\beta<1$ and $0<\gamma<1$, we have that $w \in A_{p}^{\beta}(\Omega)$ if and only if $w \in A_{p}^{\gamma}(\Omega)$. In view of this fact, we say that $w \in A_{p}^{l o c}(\Omega)$ if $w \in A_{p}^{\beta}(\Omega)$ for some $0<\beta<1$. We will call a ball satisfying this condition for some $\beta$, admissible. To simplify notation, we will use the usual notation $w(S)=\int_{S} w(x) d x$.

Proposition 3.1. Let $w$ be a weight. Then, $w \in A_{p}^{l o c}(\Omega)$ if and only if

$$
\left(f_{B}\right)^{p} \leq \frac{C}{w(B)} \int_{B} f^{p} w d x
$$

for all $f$ nonnegative and for all admissible ball $B$.

Proof. The proof follows as in the case of the $A_{p}$ weights given in [S, Chapter V, Section 1.4] and [D, Theorem 7.1] using the results in [HSV] and considering admissible balls.

Let $f \in L_{w}^{p}(\Omega)$ and consider the homogeneous boundary value problem

$$
\begin{cases}(-\Delta)^{m} u=f & \text { in } \Omega \\ \left(\frac{\partial}{\partial \nu}\right)^{j} u=0 & \text { on } \partial \Omega, \quad 1 \leq j \leq m-1\end{cases}
$$


then

$$
u(x)=\int_{\Omega} G_{m}(x, y) f(y) d y
$$

where $G_{m}(x, y)$ is the Green function of the operator $(-\Delta)^{m}$ in $\Omega$. It is well known that

$$
G_{m}(x, y)=\Gamma(x-y)+h(x, y)
$$

where $\Gamma$ is the fundamental solution given by

$$
\left\{\begin{array}{lr}
c_{m, n}|x|^{2 m-n} & n \text { odd, or } n \text { even and } n>2 m \\
c_{m, n}|x|^{2 m-n} \log |x| & n \text { even and } n \leq 2 m
\end{array}\right.
$$

and, for each $y \in \Omega, h(x, y)$ satisfies

$$
\begin{cases}\left(-\Delta_{x}\right)^{m} h(x, y)=0 & x \in \Omega \\ \left(\frac{\partial}{\partial \nu}\right)^{j} h(x, y)=-\left(\frac{\partial}{\partial \nu}\right)^{j} \Gamma(x-y) & x \in \partial \Omega, 0 \leq j \leq m-1 .\end{cases}
$$

Let us recall that, for $|\gamma|=2 m$, a standard argument yields

$$
D_{x}^{\gamma} \int_{\Omega} \Gamma(x-y) f(y) d y=\lim _{\varepsilon \rightarrow 0} \int_{|x-y|>\epsilon} D^{\gamma} \Gamma(x-y) f(y) d y+c(x) f(x)
$$

where $c$ is a bounded function.

We will use the ideas given in [S, Chapter V, Section 4.6].

Lemma 3.2. For $|\gamma|=2 m$ we have

1. There exists $u_{0} \in \mathbb{R}^{n}$ with $\left|u_{0}\right|=1$ and a constant $C_{0}$ such that, for all positive numbers $t$,

$$
\left|D^{\gamma} \Gamma\left(t u_{0}\right)\right| \geq C_{0} t^{-n}
$$

2. There exists $t_{0}$ such that if $u=t_{0} u_{0}$ and $|v| \leq 2$ then for all $0 \neq r \in \mathbb{R}$,

$$
\left|D^{\gamma} \Gamma(r(u+v))-D^{\gamma} \Gamma(r u)\right| \leq \frac{1}{2}\left|D^{\gamma} \Gamma(r u)\right| .
$$

Proof. One can check that $D^{\gamma} \Gamma$ is homogeneous of degree $-n$ and not identically zero. Then, there exists $u_{0} \in \mathbb{R}^{n}$ with $\left|u_{0}\right|=1$ such that $\left|D^{\gamma} \Gamma\left(u_{0}\right)\right|=$ : $C_{0}>0$, and therefore, (1) follows from the homogeneity.

To prove (2) we observe first that, by homogeneity, it is enough to show that the statement holds for $r=1$. 
Take $v \in \mathbb{R}^{n}$ satisfying $|v| \leq k|u|$ with $k \leq \frac{1}{2}$ to be chosen below. Then, for some $\xi$ in the segment joining $u$ and $u+v$,

$$
\left|D^{\gamma} \Gamma(u+v)-D^{\gamma} \Gamma(u)\right| \leq\left|\nabla D^{\gamma} \Gamma(\xi)\right||v| \leq C|\xi|^{-n-1}|v|
$$

and using $|u| \leq 2|\xi|,|v| \leq k|u|$ and (1) we obtain,

$$
\left|D^{\gamma} \Gamma(u+v)-D^{\gamma} \Gamma(u)\right| \leq C_{1} k|u|^{-n} \leq \frac{C_{1} k}{C_{0}}\left|D^{\gamma} \Gamma(u)\right| .
$$

Consequently it is enough to choose $k$ such that $\frac{C_{1}}{C_{0}} k \leq \frac{1}{2}$. Now, since $|v| \leq 2$, if we choose $t_{0}=\frac{2}{k}$ our hypothesis $|v| \leq k|u|$ is verified and the proof is concluded.

The following result is proved in ([DST2, Proposition 3.3]).

Lemma 3.3. There exists a constant $C$ such that, for $|x-y| \leq d(x)$,

$$
\left|D_{x}^{\gamma} h(x, y)\right| \leq C d(x)^{-n}
$$

Now, using the representation (3.3) and (3.4), we have

$$
D^{\gamma} u(x)=T_{\gamma} f(x)+c(x) u(x)
$$

where $T_{\gamma}$ is defined by

$$
T_{\gamma} f(x)=\lim _{\varepsilon \rightarrow 0} \int_{|x-y|>\epsilon} D^{\gamma} \Gamma(x-y) f(y) d y+\int_{\Omega} D_{x}^{\gamma} h(x, y) f(y) d y .
$$

We can now prove the main result of this section.

Theorem 3.4. Let $u$ be the solution of (3.2). If $w$ is a weight such that the following a priori estimate

$$
\|u\|_{W_{w}^{2 m, p}(\Omega)} \leq C\|f\|_{L_{w}^{p}(\Omega)}
$$

holds, then $w \in A_{p}^{l o c}(\Omega)$.

Proof. In view of (3.5), since $c$ is a bounded function, it is enough to prove that, if for any $\gamma$ such that $|\gamma|=2 m$,

$$
\left\|T_{\gamma} f\right\|_{L_{w}^{p}(\Omega)} \leq C\|f\|_{L_{w}^{p}(\Omega)}
$$

then $w \in A_{p}^{l o c}(\Omega)$.

We write $T_{\gamma}=T_{1}+T_{2}$ where

$$
T_{1} f(x)=\lim _{\varepsilon \rightarrow 0} \int_{|x-y|>\epsilon} D^{\gamma} \Gamma(x-y) f(y) d y
$$


and

$$
T_{2} f(x)=\int_{\Omega} D_{x}^{\gamma} h(x, y) f(y) d y .
$$

Consider an admissible ball $B=B(\bar{x}, r)$, i.e., $2 r<\beta \operatorname{dist}(B, \partial \Omega)$, with $\beta$ to be determined later and $B^{\prime}:=B(\bar{x}+r u, r)$, with $u=t_{0} u_{0}$ as in Lemma 3.2. We will see that $\beta$ can be taken so that $B^{\prime}$ is also admissible. Let $x \in B^{\prime}$ and $z \in \partial \Omega$ then $x=\bar{x}+r u+r x^{\prime}$ with $\left|x^{\prime}\right| \leq 1$,

$$
\begin{aligned}
|x-z| & \geq|\bar{x}-z|-r\left|u+x^{\prime}\right| \\
& \geq \operatorname{dist}(B, \partial \Omega)-r\left|u+x^{\prime}\right| \\
& \geq \frac{2 r}{\beta}-r\left|u+x^{\prime}\right| \\
& \geq 2 r\left(\frac{1}{\beta}-\frac{t_{0}+1}{2}\right),
\end{aligned}
$$

so, taking $\beta$ satisfying $\frac{1}{\beta}-\frac{\left(t_{0}+1\right)}{2}>1$, i.e. $\beta<\frac{2}{t_{0}+3}$ and $\frac{1}{\alpha}=\frac{1}{\beta}-\frac{\left(t_{0}+1\right)}{2}$, we have $2 r<\alpha \operatorname{dist}\left(B^{\prime}, \partial \Omega\right)$.

Now, we will show that for $x \in B^{\prime}$ and $y \in B, D^{\gamma} \Gamma(x-y)$ has constant sign. Indeed, writing $x=\bar{x}+r u+r x^{\prime}$ and $y=\bar{x}+r y^{\prime}$ with $\left|x^{\prime}\right|,\left|y^{\prime}\right| \leq 1$, we have $x-y=r u+r v$ with $|v|=\left|x^{\prime}-y^{\prime}\right| \leq 2$. Then, by (2) from Lemma 3.2 we obtain, for $D^{\gamma} \Gamma(r u)>0$,

$$
\frac{1}{2} D^{\gamma} \Gamma(r u) \leq D^{\gamma} \Gamma(x-y) \leq \frac{3}{2} D^{\gamma} \Gamma(r u),
$$

while for $D^{\gamma} \Gamma(r u)<0$

$$
\frac{3}{2} D^{\gamma} \Gamma(r u) \leq D^{\gamma} \Gamma(x-y) \leq \frac{1}{2} D^{\gamma} \Gamma(r u) .
$$

Consequently, taking $f \in C_{0}^{\infty}(B)$ positive, we have

$$
\left|T_{1} f(x)\right|=\int_{B}\left|D^{\gamma} \Gamma(x-y)\right| f(y) d y .
$$

and moreover, using (3.6), (3.7) and property (1) of Lemma 3.2,

$$
\left|T_{1} f(x)\right| \geq \frac{1}{2} \int_{B}\left|D^{\gamma} \Gamma(r u)\right| f(y) d y \geq C_{0}\left(r t_{0}\right)^{-n} \int_{B} f(y) d y=C_{1} f_{B} .
$$

with a constant $C_{1}$ depending only on $t_{0}, C_{0}$ and $n$.

On the other hand, in order to bound $\left|T_{2} f(x)\right|$ we use Lemma 3.3. We require $|x-y| \leq d(x)$, but,

$$
|x-y|=\left|r u+r\left(x^{\prime}-y^{\prime}\right)\right| \leq r\left(|u|+\left|x^{\prime}-y^{\prime}\right|\right) \leq r\left(t_{0}+2\right)<\frac{\alpha}{2} d(x)\left(t_{0}+2\right)
$$


and then, we need $\frac{\alpha}{2}\left(t_{0}+2\right)<1$ or equivalently $\beta<\frac{2}{2 t_{0}+3}$. Now, we have that

$$
\begin{aligned}
\left|T_{2} f(x)\right| \leq \int_{B}\left|D_{x}^{\gamma} h(x, y)\right| f(y) d y \leq C \int_{B} d(x)^{-n} f(y) d y & \leq C(2 r)^{-n} \alpha^{n} \int_{B} f(y) d y \\
& =C_{2} \alpha^{n} f_{B} .
\end{aligned}
$$

where $C$ is the constant appearing in Lemma 3.3 and $C_{2}$ depends on $n$ and $C$.

Summing up we have

$$
\left|T_{\gamma} f(x)\right| \geq\left(C_{1}-C_{2} \alpha^{n}\right) f_{B}
$$

In order to have $C_{1}-C_{2} \alpha^{n}>0$ it is enough that $\beta<\frac{1}{\frac{t_{0}+1}{2}+\left(\frac{C_{2}}{C_{1}}\right)^{\frac{1}{n}}}$.

Taking into account the other conditions for $\beta$ we choose $\beta<\min \left\{\frac{2}{2 t_{0}+3}, \frac{1}{\frac{t_{0}+1}{2}+\left(\frac{C_{2}}{C_{1}}\right)^{\frac{1}{n}}}\right\}$, and then, we obtain $f_{B} \leq C\left|T_{\gamma} f(x)\right|$, for any $x \in B^{\prime}$. Therefore,

$$
\int_{B^{\prime}} f_{B}^{p} w(x) d x \leq C \int_{B^{\prime}}\left|T_{\gamma} f(x)\right|^{p} w(x) d x \leq C \int_{\Omega}\left|T_{\gamma} f(x)\right|^{p} w(x) d x
$$

and consequently, applying the continuity of $T_{\gamma}$ in $L_{w}^{p}$, we obtain

$$
\left(f_{B}\right)^{p} w\left(B^{\prime}\right) \leq C \int_{B} f(x)^{p} w(x) d x .
$$

Analogously, changing roles of $B$ and $B^{\prime}$ and taking $f$ with support in $B^{\prime}$, it follows that

$$
\left(f_{B^{\prime}}\right)^{p} w(B) \leq C \int_{B^{\prime}} f(x)^{p} w(x) d x .
$$

provided that $C_{1}-C_{2} \beta^{n}>0$. But this inequality holds for our previous election of $\beta$ because $\beta<\alpha$.

By a passage to the limit, the inequalities (3.8) and (3.9) extend to any non-negative function $f$ supported in $B$ or $B^{\prime}$ respectively. If we consider $f=\chi_{B^{\prime}}$ in (3.9) we obtain

$$
w(B) \leq C w\left(B^{\prime}\right)
$$

Using this in (3.8) we get

$$
\left(f_{B}\right)^{p} w(B) \leq C \int_{B} f(x)^{p} w(x) d x
$$

but, by Proposition 3.1, this means that $w \in A_{p}^{\text {loc }}(\Omega)$. 


\section{Acknowledgements}

This research was supported by Universidad de Buenos Aires under grant 20020120100050 and by ANPCyT, Argentina, under grant PICT 2014-1771. The results of this paper are part of the doctoral thesis of the first author which was done under a fellowship granted by CONICET, Argentina. We thank the anonymous referee for helpful comments that led us to improve the previous version of this work.

\section{References}

[ADN] S. Agmon, A. Douglis and L. Nirenberg, Estimates near the boundary for solutions of elliptic partial differential equations satisfying general boundary conditions. I, Comm. Pure Appl. Math 12 (1959), 623727.

[DRS] L. Diening, M. Ružicka, K. Schumacher, A decomposition technique for John domains, Ann. Acad. Sci. Fenn. Math 35 (2010), 87-114.

[D] J. Duoandikoetxea, Fourier Analysis, Graduate Studies in Mathematics 29 AMS, Providence, RI, (2001).

[DST1] R.G. Durán, M. Sanmartino and M. Toschi, Weighted a priori estimates for Poisson equation, Indiana University Math. Journal. 57, (2008) , 3463-3478.

[DST2] R.G. Durán, M. Sanmartino and M. Toschi, Weighted a priori estimates for the solution of the homogeneous Dirichlet problem for powers of the Laplacian Operator, Analysis in Theory and Applications, 26(4),(2010) 339-349.

[FS] C. Fefferman and E.M. Stein, $H_{p}$ spaces of several variables Acta Math. 129 (1972), no. 3-4, 137-193.

[K] J.P.Krasovskii, Isolation of the singularity in Green's function, (Russian) Izv.Akad. Nauk SSSR Ser. Mat. 31 (1967) 977-1010

[M] B. Muckenhoupt, Weighted norm inequalities for the Hardy maximal function, Trans. Amer. Math. Soc. 165 (1972), 207-226.

[HSV] E. Harboure, O. Salinas and B. Viviani, Local maximal function and weights in a general setting, Math. Ann. 358 (2014), no. 3-4, 609-628. 
[S] E.M. Stein, Harmonic Analysis: Real variable-methods, ortogonality, and oscillatory integrals, Princeton Univ. Press, 1993. 